\title{
Characterization of Freestream Disturbances in Conventional Hypersonic Wind Tunnels
}

\author{
Lian Duan ${ }^{1}$ \\ Missouri University of Science and Technology, Rolla, MO, 65409, USA \\ Meelan M. Choudhari ${ }^{2}$ and Amanda $\mathrm{Chou}^{3}$ \\ NASA Langley Research Center, Hampton, VA 23683, USA \\ Federico Munoz ${ }^{4}$, Syed Raza Christopher $\mathrm{Ali}^{4}$, and Rolf Radespiel ${ }^{5}$ \\ Institute of Fluid Mechanics, TU Braunschweig, Hermann-Blenk-Str. 37, 38108 Braunschweig, Germany \\ Thomas Schilden ${ }^{6}$ and Wolfgang Schröder ${ }^{7}$ \\ Institute of Aerodynamics, RWTH Aachen University, Wüllnerstr. 5a, 52062, Germany \\ Eric C. Marineau ${ }^{8}$ \\ AEDC White Oak, Silver Spring, MD, 20903, USA \\ Katya M. Casper ${ }^{9}$ \\ Sandia National Laboratories, Albuquerque, NM 87185, USA \\ Ross S. Chaudhry ${ }^{10}$ and Graham V. Candler ${ }^{11}$ \\ University of Minnesota, Minneapolis, MN, 55455, USA \\ Kathryn A. Gray ${ }^{10}$, Cameron J. Sweeney ${ }^{12}$ and Steven P. Schneider ${ }^{13}$ \\ Purdue University, West Lafayette, Indiana 47907, USA
}

\begin{abstract}
While low disturbance ("quiet") hypersonic wind tunnels are believed to provide more reliable extrapolation of boundary layer transition behavior from ground to flight, the presently available quiet facilities are limited to Mach 6, moderate Reynolds numbers, low freestream enthalpy, and subscale models. As a result, only conventional ("noisy") wind tunnels can reproduce both Reynolds numbers and enthalpies of hypersonic flight configurations, and must therefore be used for flight vehicle test and evaluation involving high Mach number, high enthalpy, and larger models. This article outlines the recent progress and achievements in the characterization of tunnel noise that have resulted from the coordinated effort within the AVT-240 specialists group on hypersonic boundary layer transition prediction. New Direct Numerical Simulation (DNS) datasets elucidate the physics of noise generation inside the turbulent nozzle wall boundary layer, characterize the
\end{abstract}

\footnotetext{
${ }^{1}$ Assistant Professor. Senior Member AIAA.

${ }^{2}$ Senior Research Scientist, Computational AeroSciences Branch, Associate Fellow AIAA.

${ }^{3}$ Research Aerospace Engineer, Flow Physics \& Control Branch, Senior Member AIAA.

${ }^{4}$ Research Assistant, Institute of Fluid Mechanics TU-Braunschweig.

${ }^{5}$ Professor, Head of Institute, Institute of Fluid Mechanics TU-Braunschweig, Associate Fellow AIAA.

${ }^{6}$ Research Scientist, Institute of Aerodynamics, RWTH Aachen University.

${ }^{7}$ Head, Institute of Aerodynamics, RWTH Aachen University.

${ }^{8}$ Chief Technologist. Senior Member AIAA.

${ }^{9}$ Senior Member of the Technical Staff. Senior Member AIAA.

${ }^{10}$ Graduate Student. Student Member AIAA.

${ }^{11}$ McKnight Presidential Professor and Russell J. Penrose Professor. Fellow AIAA.

${ }^{12}$ Graduate Student. Student Member AIAA (Present affiliation: Science Applications International Corporation).

${ }^{13}$ Professor. Associate Fellow AIAA.
} 
spatiotemporal structure of the freestream noise, and account for the propagation and transfer of the freestream disturbances to a pitot-mounted sensor. The new experimental measurements cover a range of conventional wind tunnels with different sizes and Mach numbers from 6 to 14 and extend the database of freestream fluctuations within the spectral range of boundary layer instability waves over commonly tested models. Prospects for applying the computational and measurement datasets for developing mechanism-based transition prediction models are discussed.

\section{Nomenclature}
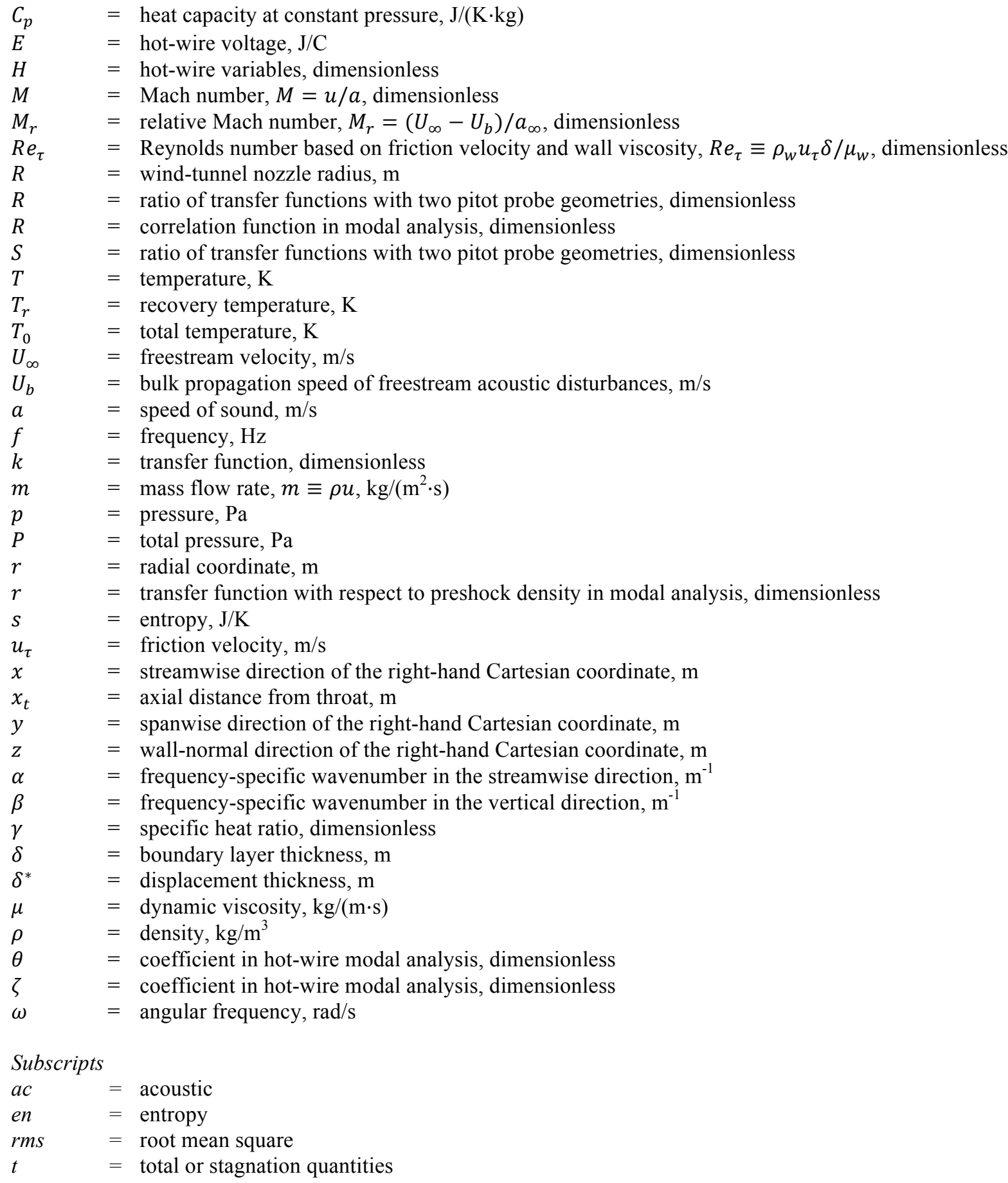


$$
\begin{array}{ll}
\text { vo } & =\text { vortical } \\
w & =\text { wall variables } \\
\infty & =\text { freestream variables } \\
0 & =\text { stagnation quantities } \\
1 & =\text { preshock quantities of a pitot probe } \\
2 & =\text { post-shock quantities of a pitot probe }
\end{array}
$$

$\begin{array}{ll}\begin{array}{ll}\text { Superscripts } \\ +\end{array} & =\text { inner wall units } \\ \overline{(\cdot)} & =\text { averaged variables } \\ (\cdot)^{\prime} & =\text { perturbation from averaged variables }\end{array}$

\section{Introduction}

Prediction of boundary-layer transition is a critical part of the design of hypersonic vehicles because of the large increase in skin-friction drag and surface heating associated with the onset of transition. Testing in conventional (noisy) wind tunnels has been an important means of characterizing and understanding the boundary-layer transition (BLT) behavior of hypersonic vehicles. Because the existing low disturbance, i.e., quiet, facilities operate only at Mach 6, moderate Reynolds numbers, fairly small sizes, and low freestream enthalpy, conventional facilities will continue to be employed for testing and evaluation of hypersonic vehicles, especially for ground testing involving other Mach numbers, higher freestream enthalpies, and larger models. To enable better use of transition data from conventional facilities and more accurate extrapolation of wind-tunnel results to flight, one needs an in-depth knowledge of the broadband disturbance environment in those facilities as well as of the interaction between the freestream disturbances with laminar boundary layers.

Freestream disturbances in conventional high-speed wind tunnels are usually composed of acoustic disturbances, vorticity disturbances and fluctuations of flow entropy. The acoustic disturbances are mainly generated within the high-speed, turbulent boundary layers along the nozzle walls and radiated into the wind tunnel test section [7]. The intensity of the acoustic disturbances increases rapidly with flow Mach number; and hence, these disturbances are likely to dominate the overall disturbance environment at Mach numbers of 2.5 or above [8-11] and can strongly affect the transition processes. Theoretical models for acoustic radiation from a supersonic boundary layer were developed by Phillips [6] and Ffowcs-Williams [12], who attributed a major cause of the acoustic radiation to eddy Mach waves from boundary layer turbulence convecting supersonically with respect to the free stream. A lack of adequate knowledge concerning the boundary layer turbulence restricted the theoretical predictions to just the intensity of the freestream acoustic fluctuations. Fluctuations of freestream vorticity and entropy stem from the flow state in the tunnel settling chamber and the subsequent changes imposed by the wind tunnel nozzle. Figure 1 gives a schematic of the origin of freestream disturbances in a supersonic/hypersonic wind tunnel.

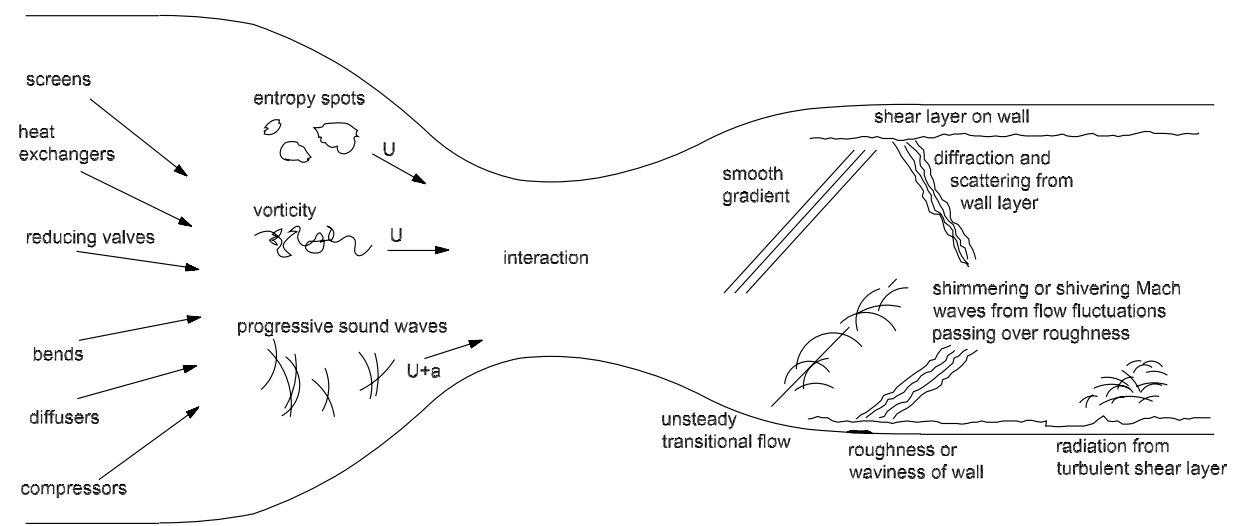

Figure 1. Schematic of freestream disturbances in supersonic/hypersonic wind tunnels (adapted from Schneider [1]). 
The freestream disturbances in conventional tunnels can impact not only the transition location, but possibly the transition mechanism as well, and their effect on transition cannot be quantified in terms of a single metric corresponding to the r.m.s. amplitude, as indicated by the measurements at Purdue University [13] and AEDC Tunnel 9 [14]. Unfortunately, existing measurements mostly provide data in terms of r.m.s. values alone, i.e., without an evaluation of the disturbance spectra up to the high frequencies observed in transitional hypersonic boundary layers. Although a number of investigators have reported measurements of freestream disturbance intensity in high-speed facilities at both supersonic and hypersonic Mach numbers (see, for example,[15-19]), these measurements are largely limited to single-point information (e.g., freestream pitot pressure fluctuations) and the measurements by Laufer $[9,20]$ still reflect one of the few datasets that are detailed enough to be suitable for comparison or model development.

Recently, new probes and new instrumentation have become available to the research community that greatly help the physical characterization of freestream disturbance levels [21,22]. The new experimental data are able to cover a spectral range of disturbances that were previously not achieved by using hot-wire probes. Progress in direct numerical simulation (DNS) as well as the rapid deployment of high-performance computing facilities across the US and around the world provide the opportunity to address the problem of the generation of acoustic disturbances at the nozzle walls and the transfer of those disturbances to the location of a pitot-mounted sensor, making the numerical rebuilding of transition experiments possible. The progress in both experimental and numerical techniques holds the potential to bridge the gap between quiet tunnels, conventional wind tunnels, and flight.

The current paper summarizes the coordinated experimental and numerical work undertaken by the NATO STO AVT-240 specialists group to characterize the freestream disturbances in conventional hypersonic ground facilities. Definitive progress has been made possible by coordinating international research efforts in this difficult but important area that will eventually enable improved ground-to-flight scalability of the laminar-turbulent transition data from conventional high-speed wind-tunnel facilities.

The paper is structured as follows. Measurements of freestream disturbances in various high-speed facilities with new probes and new instrumentation as well as closely related DNS are outlined in Section II. Section III presents DNS studies for synthesizing the naturally occurring, random acoustic disturbances induced by tunnel wall turbulence. Section IV is focused on numerical rebuilding of tunnel freestream disturbances from the measurement of intrusive probes. Section V outlines a summary of the overall findings and a general vision for future research.

\section{Tunnel Freestream Disturbance Measurement}

Measurements of freestream disturbances in multiple high-speed facilities at hypersonic Mach numbers have been reported with new probes and new instrumentation. Tunnel noise has now been measured with new, fast PCB132 pressure sensors that allow noise characterization up to $1 \mathrm{MHz}$, which is above the second-mode frequencies in most tunnels. Enabled by the coordinated international research effort, a comparison of tunnel noise measurements in different hypersonic wind tunnels is made that allows for cross-validation and, more importantly, sheds light on whether tunnel-to-tunnel noise variation follows a relatively simple pattern as suggested by Pate's correlation $[23,24]$.

Figure 2 shows the power spectral density (PSD) of freestream pitot-pressure fluctuations measured in multiple high-speed facilities. The measurement data covers a wide range of tunnel conditions, including the Hypersonic Ludwieg Tube Braunschweig (HLB), the Purdue Boeing/AFOSR Mach-6 Quiet Tunnel (BAM6QT), the NASA 20Inch Mach 6, the Sandia Hypersonic Wind Tunnel at Mach 8 (HWT-8), and the AEDC Tunnel 9; these wind tunnels generate freestream Mach numbers ranging from Mach 6 to 14. The PSD are computed with pressure signals measured by Kulite ${ }^{\circledR}$ sensors in the low-frequency range up to $f \approx 20 \mathrm{kHz}$ and by PCB PIEZOTRONICS, Inc. sensors in the high-frequency range up to $f \approx 1 \mathrm{MHz}$. The frequency range measured by the $\mathrm{PCB}^{\circledR}$ sensors covers that of second-mode waves. For all the cases, the freestream pitot-pressure spectrum has a similar rate of spectral roll-off at high frequencies. A spectral slope of $f^{-3.5}$ provides a good fit to the data over the second-mode frequency range. The data suggests that the freestream disturbance spectrum relevant to second-mode-dominated boundary-layer transition may be modeled with a constant-slope model of $\Phi \sim f^{-m}$. Such a model has been used by Marineau [25] who proposed a new amplitude method for predicting second-mode-dominated boundary-layer transition in hypersonic wind tunnels. Marineau's amplitude method was found to reduce the error between the measured and predicted start of transition, compared with predictions based on a constant transition $\mathrm{N}$ factor [25]. Because of measurement difficulties at high frequencies and the nontrivial transfer function associated with measurement probes, physics-based transition models of this type cannot rely solely upon the measurement database and synergistic numerical simulations are also important in developing more accurate and reliable models for the 
tunnel disturbance environment. Direct computations of acoustic freestream disturbances are discussed in Section III below, whereas the recovery of actual freestream disturbances from probe measurements is addressed in Section IV.

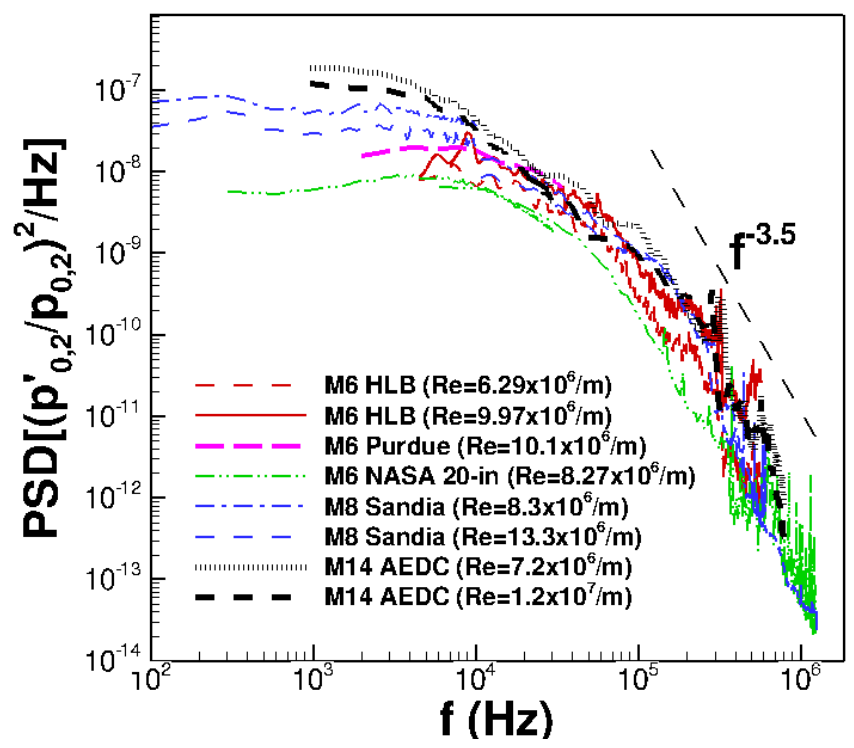

Figure 2. Power spectral density of freestream pitot-pressure fluctuations measured in multiple high-speed facilities. The pressure transducer models and the freestream locations for pitotpressure measurements are listed as follows: HLB Mach $6\left(\mathrm{PCB}^{\circledR}: x_{t}=2.69 \mathrm{~m}, r=0.1 \mathrm{~m}\right)$; Purdue BAM6QT-noisy (Kulite ${ }^{\circledR}: x_{t}=2.3368 \mathrm{~m}, r=0 \mathrm{~m}$ ); NASA 20-Inch Mach 6 (Kulite ${ }^{\circledR} \&$ PCB $\left.^{\circledR}: x_{t}=2.25 \mathrm{~m}, r=0.09042 \mathrm{~m}\right)$; Sandia HWT-8 $\left(\right.$ Kulite $^{\circledR}$ \& PCB $^{\circledR}: x_{t}=2.3363 \mathrm{~m}, r=0$ m); AEDC Tunnel 9 Mach $14\left(\mathrm{PCB}^{\circledR}: x_{t}=13.97 \mathrm{~m}, r=0.38 \mathrm{~m}\right)$.

\section{Direct Simulation of Tunnel Acoustic Disturbances}

An important component of the recent progress in tunnel-noise characterization is to use DNS for synthesizing the naturally occurring, random acoustic disturbances created within the tunnel wall turbulence. Given that in unheated conventional hypersonic tunnels with adequate flow conditioning, the freestream disturbance environment is dominated by acoustic radiation from tunnel-wall turbulent boundary layers (TBL) [8-11] (Fig. 3), DNS can be used to resolve both the tunnel-wall boundary layer and the near field of acoustic fluctuations radiated by the boundary layer, thus allowing access to tunnel-noise quantities that are difficult to obtain otherwise and for clarifying the physics of noise generation in conventional hypersonic facilities.

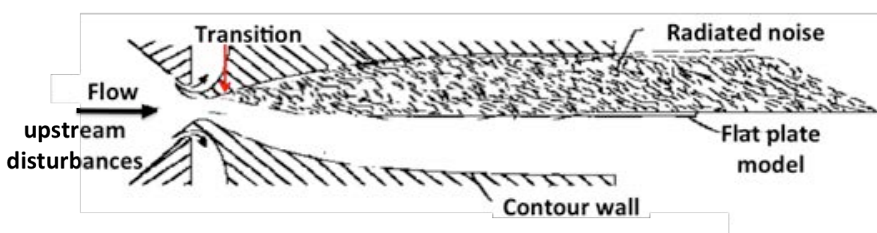

(a)

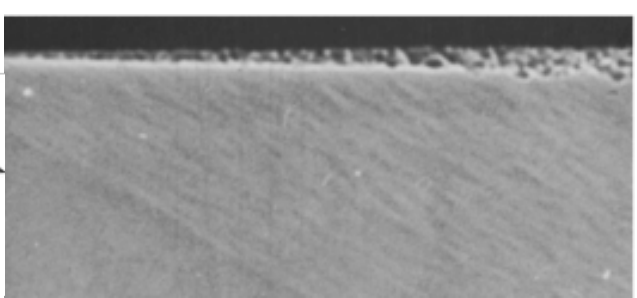

(b)

Figure 3. (a) Sketch of the test core of a Mach 3.5 wind tunnel and radiated noise from the tunnelwall turbulent boundary layer (adapted from Beckwith \& Miller [2]); (b) Experimental image of a shadowgraph for a Mach 3.5 boundary layer, which shows that the acoustic nearfield in the freestream region consists of randomly spaced wavepackets (courtesy of NASA Langley). 
The research teams of Missouri S\&T and NASA Langley developed a systematic strategy that first established the feasibility of DNS in the context of a canonical, single-wall boundary layer (Fig. 4) across a range of Mach numbers $[3,5,26-28]$. The DNS of acoustic radiation from a single-wall boundary layer circumvented the difficulties associated with the reflection/reverberation of acoustic waves from all sides of a wind tunnel nozzle and, thus, helped clarify the physical process of acoustic noise generation. The physical realism and accuracy of the DNS flow fields have been established by comparison with existing experimental results at similar flow conditions. In particular, the coordinated experimental and numerical work undertaken by the international specialists group has led to the first successful comparison between numerical predictions and wind tunnel measurements of surface pressure fluctuations underneath a hypersonic TBL at (nominal) Mach numbers of 6, 8, and 14 (Fig. 5). Very good agreement of wall-pressure PSD between the DNS and the nozzle-wall measurements has been achieved at Mach 6 and 8. The DNS-predicted PSD deviates from the measured PSD at low frequencies for the Mach 14 case. The large disparity in Karman Reynolds numbers during the experiment and the DNS may have contributed to the discrepancy in wall pressure PSD; however, further work is necessary to identify the precise reasons and to resolve the differences at low frequencies. The cause of the spurious peak at $f \approx 40 \mathrm{kHz}$ in the wall-pressure PSD measured at Mach 14 is unknown and is currently under investigation.

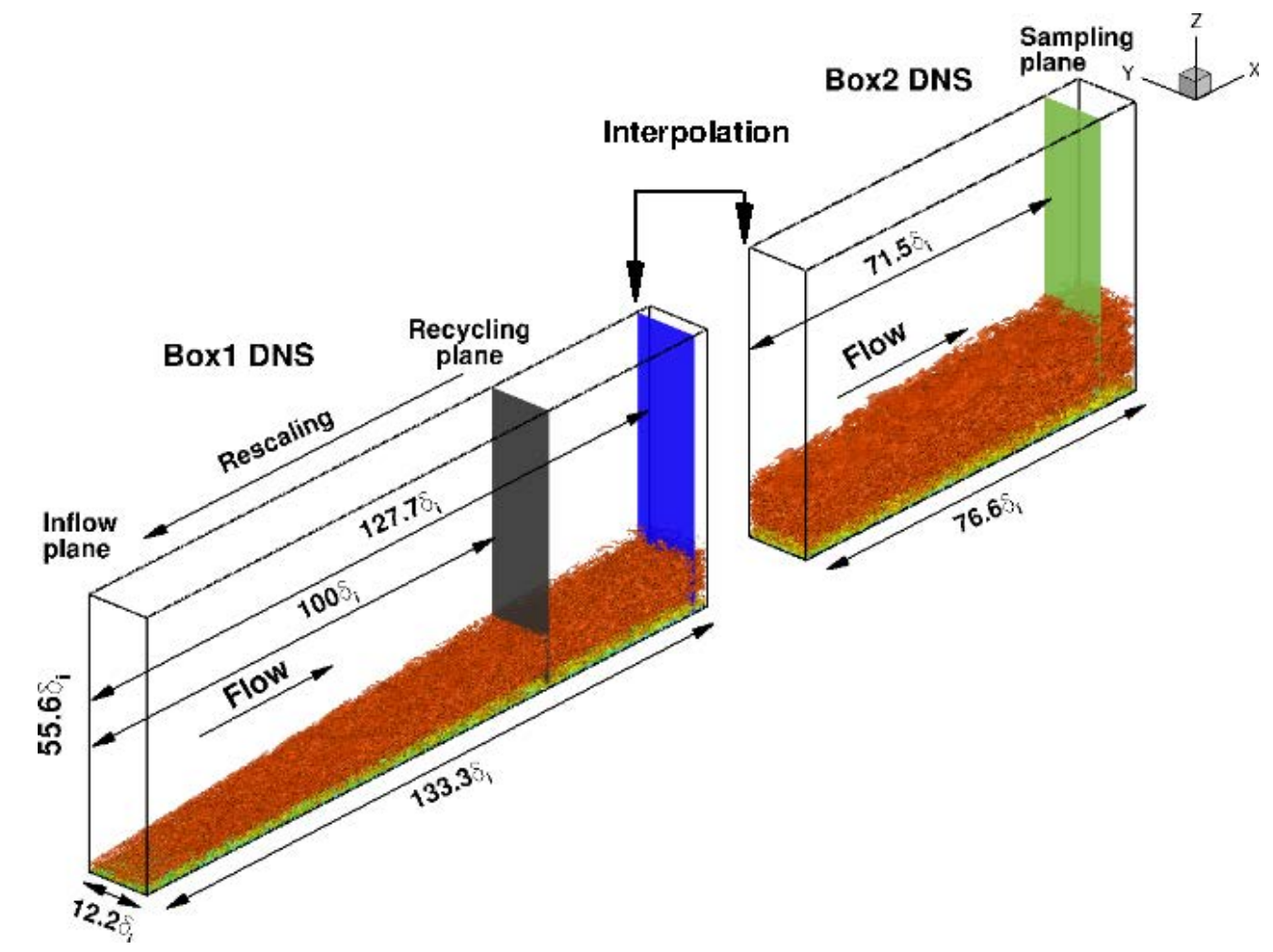

Figure 4. Computational domain and simulation setup for DNS of a Mach 14 turbulent boundary layer with flow conditions representative of the nozzle exit of AEDC Tunnel 9 (from Fig. 1 [5]). 


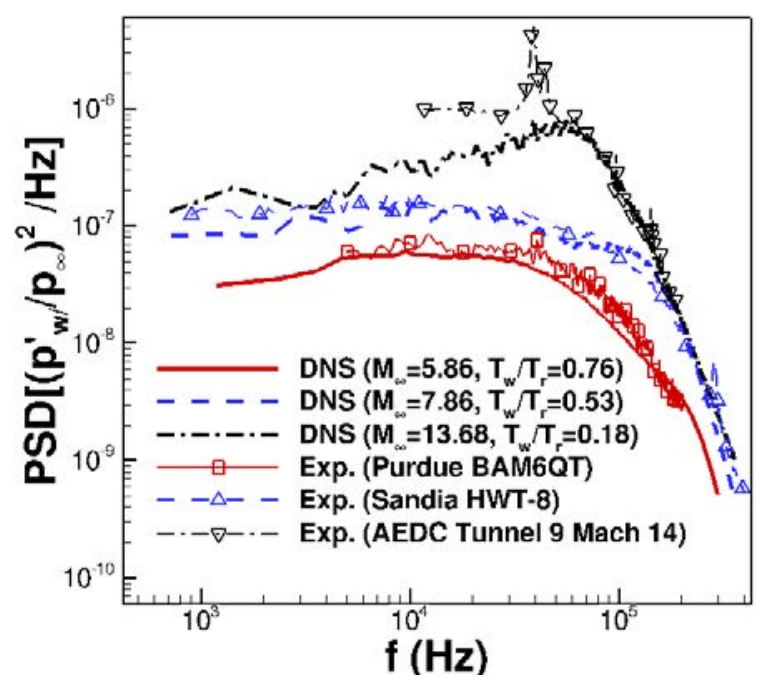

Figure 5. Comparison between numerical predictions and wind tunnel measurements of surface pressure fluctuations underneath a hypersonic TBL, with the experimental data measured on the nozzle walls of Purdue BAM6QT, Sandia HWT-8, and AEDC Tunnel 9. The pressure transducer models and the axial locations for surface-pressure measurements are listed as follows: Purdue BAM6QT $\left(\right.$ PCB $^{\circledR}: x_{t}=$ $\left.2.679 \mathrm{~m} ; \mathrm{R} e=9.69 \times 10^{6} / \mathrm{m}\right) ;$ Sandia HWT-8 $\left(\right.$ Kulite $^{\circledR}: x_{t}=2.2024 \mathrm{~m} \&$ PCB $^{\circledR}: x_{t}=2.3459 \mathrm{~m}$; $\left.R e=8.3 \times 10^{6} / \mathrm{m}\right) ;$ AEDC Tunnel 9 Mach $14\left(\mathrm{PCB}^{\circledR}: x_{t}=11.83 \mathrm{~m} ; \operatorname{Re}=7.2 \times 10^{6} / \mathrm{m}\right)$.

The DNS database has provided access to tunnel-noise quantities that are difficult to obtain otherwise, including high-frequency PSD (Fig. 6a), propagation speed (Fig. 6b), wave angle (Fig. 7a), spatial and temporal correlations (Fig. 7b), and acoustic sources (Fig. 8). DNS showed that tunnel noise consists of a field of broadband, stochastic acoustic waves that have a finite spatiotemporal coherence and propagate at oblique angles to the free stream, rather than as a deterministic train of time harmonic, planar waves as commonly assumed in receptivity studies; the acoustic sources that give rise to the pressure fluctuations in the free stream are located mostly in the inner layer of the tunnel-wall turbulent boundary layer and are strongly influenced by wall cooling, as seen from the differences in peak source location in Fig. 8 (see Ref. [3] for an explanation of this behavior); the inclination angle of the acoustic wave front is similar to the Mach angle for the relative Mach number $M_{r}$ (between the sources and free stream). The findings are consistent with the classic theory of 'eddy Mach-wave radiation' and the early measurements of freestream noise by Laufer [9] during his unique wind tunnel experiments.

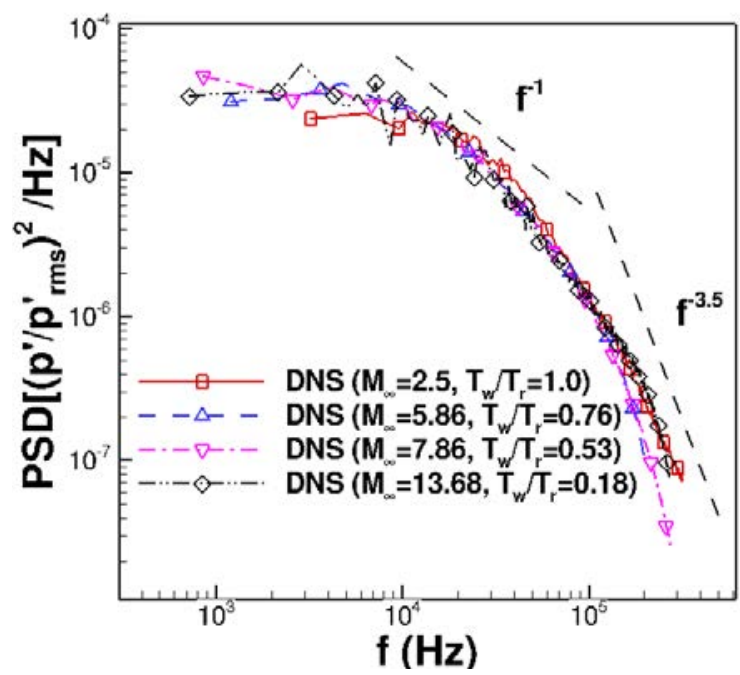

(a)

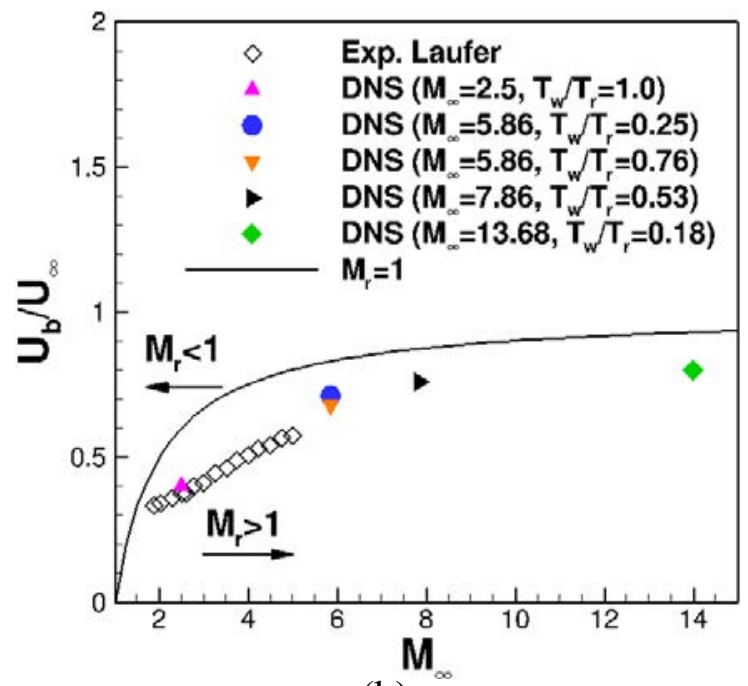

(b)

Figure 6. (a) Power spectral density and (b) bulk propagation speed of the freestream acoustic pressure fluctuations as a function of the freestream Mach number. The bulk propagation speed is estimated as the ratio between the streamwise spatial distance between probe pairs, and the time lags corresponding to the peak of the cross-correlation curve. 


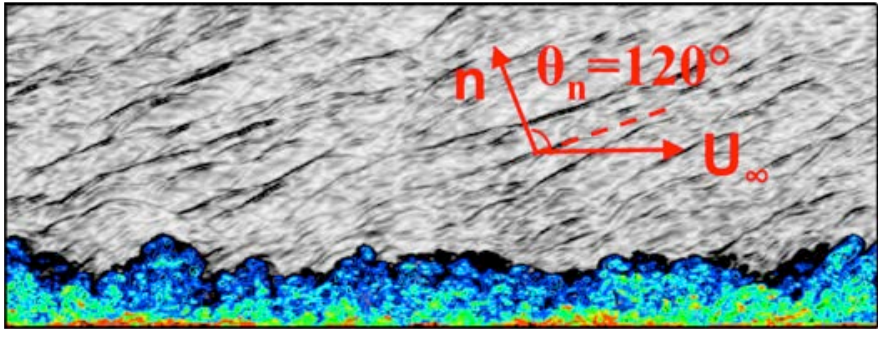

(a)

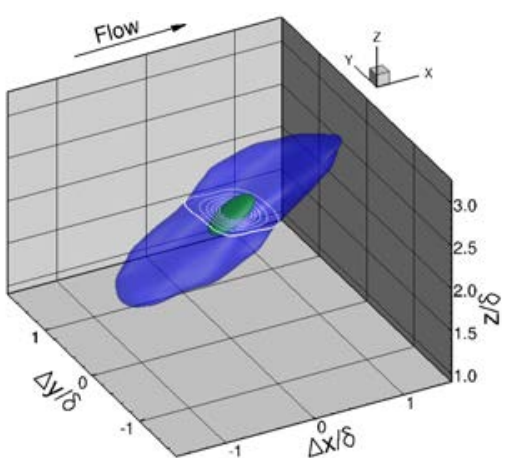

(b)

Figure 7. Freestream acoustic structures radiated from a Mach 5.86 turbulent boundary layer at a wall-to-recovery temperature ratio of $T_{w} / T_{r}=0.25$ [3]. (a) Instantaneous flow visualization, with the freestream acoustic wave front visualized by the grey contours of the density gradient. (b) Three-dimensional isosurfaces of the spatial correlation coefficient to illustrate statistically significant 3-D acoustic structures in the free stream.

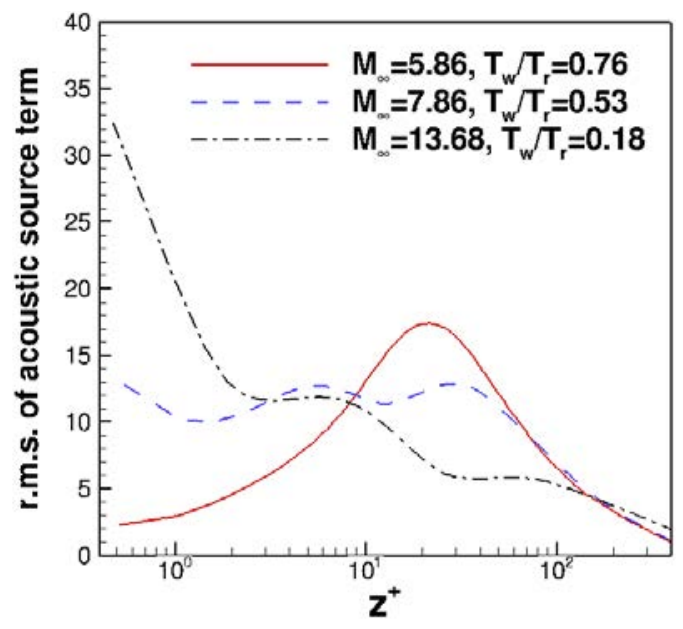

Figure 8. Acoustic source terms, $\frac{\partial u_{i}}{\partial x_{j}} \frac{\partial u_{j}}{\partial x_{i}}$, of hypersonic turbulent boundary layers that give rise

to the acoustic pressure fluctuations in the free stream. The r.m.s. of the source terms are normalized by $\delta^{2} / U_{\infty}^{2}$, and $z^{+}$is the wall-normal distance normalization by local viscous length. The acoustic source terms are defined according to the acoustic analogy by Phillips [6].

After the feasibility of the DNS was established in the context of a single flat wall, the DNS conditions were extended to those within a confined enclosure to mimic the typical case of multiple tunnel walls in an experiment. DNS were carried out in stages corresponding to increasing complexity and computational cost, progressing from internal noise within a double-wall (channel) configuration [29] to an axisymmetric cylinder [30], and finally, a fullscale nozzle of a hypersonic wind tunnel (Fig. 9). These simulations have shed light on the effect of geometry on the noise field and, in future, would help enable practical applications of the simulation data for freestream disturbances in the context of actual wind-tunnel experiments. Detailed results of DNS with multiple tunnel walls were reported in Ref. [4, 29, 30].

The most recent outcome of these DNS studies was the simulation of acoustic disturbances within the nozzle of a Mach 6 Hypersonic Ludwieg Tube, Braunschweig (HLB), at the Technical University of Braunschweig (TUB), at actual flow conditions relevant to transition measurement. As seen at the top of Fig. 9, the HLB configuration starts from the storage tube ahead of the nozzle throat, which is located at $x \approx 1.41 \mathrm{~m}$. The nozzle part spans from $x \approx 1.41 \mathrm{~m}$ to $x \approx 3.8 \mathrm{~m}$, followed by the test section region. The DNS domain starts slightly downstream of the nozzle throat at $x \approx 2.0 \mathrm{~m}$ with a local freestream Mach number of $M_{\infty}=3.84$ and ends at the nozzle exit at $x \approx 3.8 \mathrm{~m}$, with a freestream Mach number of $M_{\infty}=5.71$. The selected DNS domain covers the origin of most of 
the acoustic sources responsible for generating freestream noise in the test section, as it includes the portion of the nozzle with high freestream Mach numbers, and, thus, with large intensity of noise radiation. The DNS inflow is extracted from a precursor Reynolds-Averaged Navier-Stokes (RANS) calculation that simulates the full-domain HLB geometry, including the storage tube, the fast acting valve, the de Laval nozzle and the test section. Good comparison was achieved for the freestream Mach number distribution along the nozzle axis among DNS, RANS, and the theory (Fig. 10).

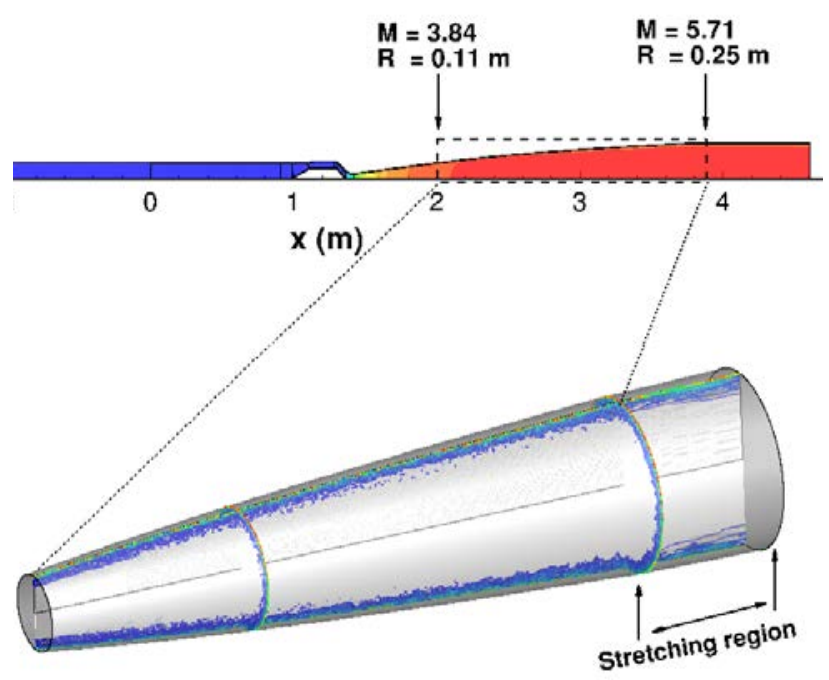

Figure 9. DNS of acoustic disturbances within the nozzle of Mach 6 Hypersonic Ludwieg Tube at the Technical University of Braunschweig (from Fig. 1 [4]).

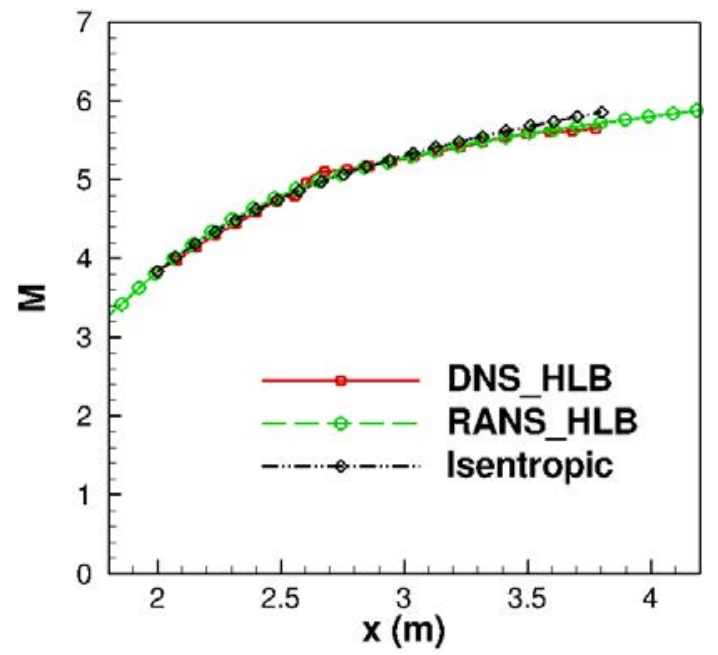

Figure 10. Comparison of Mach number distribution along the nozzle axis among DNS, RANS, and the theory (from Fig. 2a [4]).

Fig. 11 shows an instantaneous visualization of the density gradient associated with the radiated acoustic field. The wave fronts exhibit a preferred orientation of $\theta \approx 31^{\circ}$ with respect to the nozzle centerline within the streamwise-radial plane. The density gradients reveal the omnidirectional origin of the acoustic field within a given cross-section of the nozzle, which adds to the stochastic nature of the wave front pattern at a given axial location. Because of the limited length of the nozzle and shallow acoustic propagation angles (with respect to the flow direction), the number of acoustic reflections from the nozzle wall that contribute to the acoustic signal at the nozzle 
exit plane is expected to be small. Thus, additional simulations are required to establish the relative contribution from those acoustic reflections, i.e., the reverberation effect.

Figure 12 shows that the r.m.s. pressure fluctuation normalized by the wall shear stress, $p_{r m s}^{\prime} / \tau_{w}$, of the nozzle plateaus in the free stream and is approximately $20 \%$ higher than that induced by the turbulent boundary layer over a single flat plate at a similar freestream Mach number. This increase in the noise intensity is believed to be caused by the combined effect of acoustic radiation arriving from different azimuthal segments of the axisymmetric nozzle wall. For both the nozzle and flat plate, spectral analysis shows a similar frequency content of pressure fluctuations (Fig. 13).

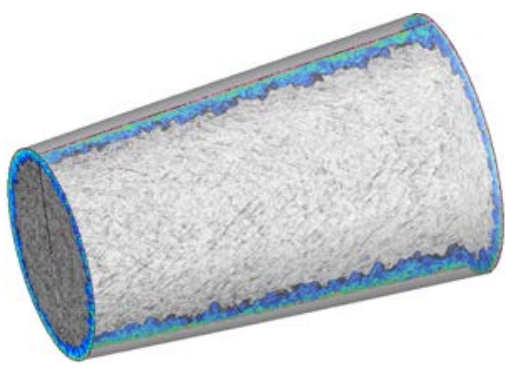

(a)

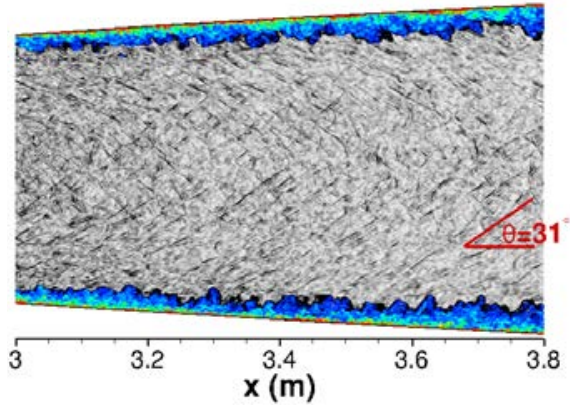

(b)

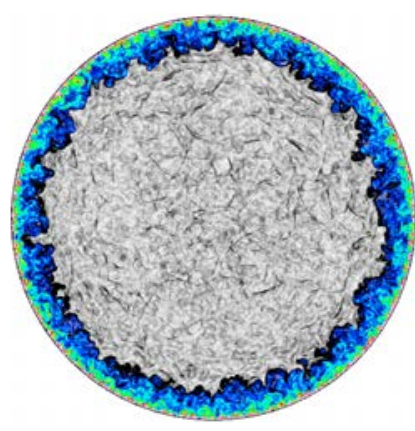

(c)

Figure 11. Numerical schlieren images (i.e., density gradient contours) of radiated acoustic waves within the nozzle of HLB. (a) Three dimensional volume, $3.0<\mathrm{x}<3.8 \mathrm{~m}$; (b) streamwise-radial plane $(3.0<x<3.8 \mathrm{~m}$ ); (c) cross section at $x=3.7 \mathrm{~m}$ (from Fig. 9 [4]).

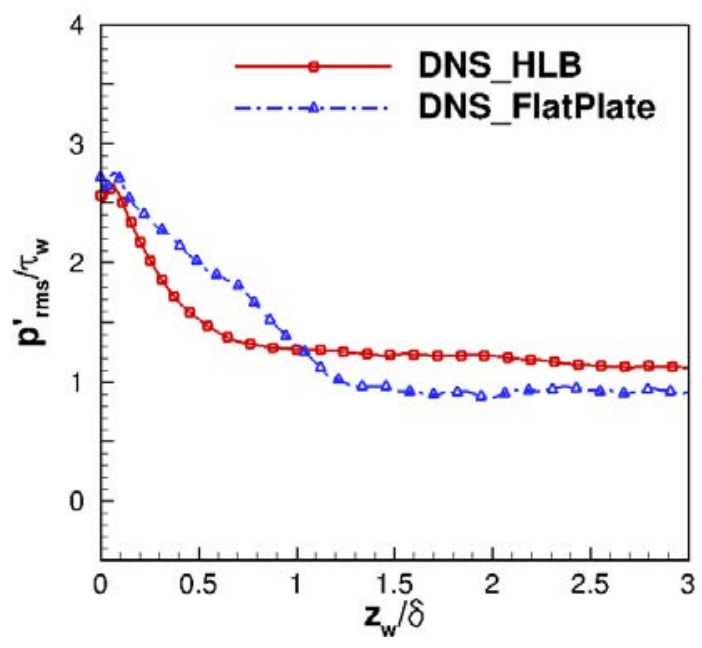

Figure 12. r.m.s. pressure fluctuation profile induced by the turbulent boundary layer over the HLB nozzle wall and a single flat plate at a similar freestream Mach number. $z_{w}$ is the wall-normal distance (from Fig. 4b [4]). 

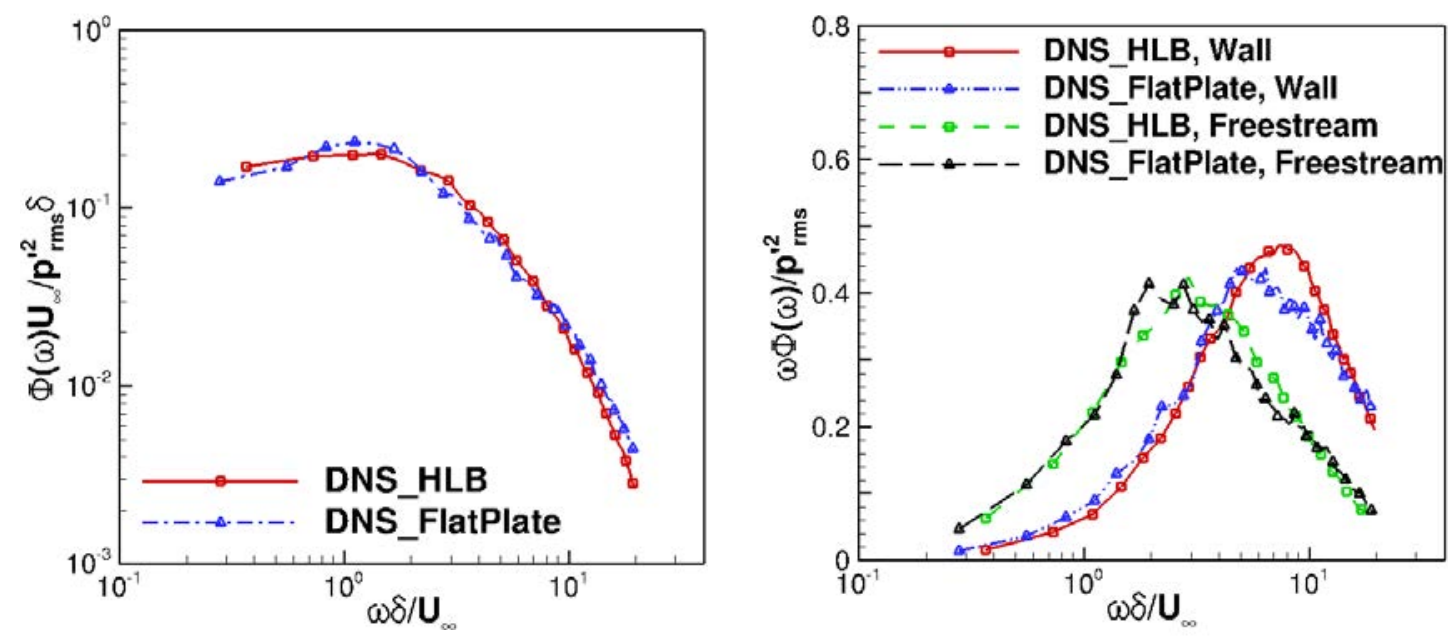

Figure 13. Power spectral density of freestream acoustic disturbances induced by a turbulent boundary layer over the HLB nozzle wall and a single flat plate at a similar freestream Mach number.

The current simulations described in this section have paved the way for improved simulations including the test section downstream of the nozzle exit. Additionally, the numerical results indicate the role of mean boundary layer quantities as important parameters in determining the characteristics of the radiated acoustic field, highlighting the need for experimental measurements of nozzle boundary layer profiles.

\section{Recovery of Tunnel Freestream Disturbances}

Another area of the internationally coordinated research effort is to recover tunnel noise spectrum from pitotprobe measurements and characterize the modal contents of tunnel freestream disturbances by modal analysis. Freestream disturbances in a hypersonic wind tunnel pass through the bow shock and the stagnation region of a pitot probe before being measured by the transducer. Therefore, the relationship between the freestream disturbance spectrum and the transducer-measured spectrum, defined as the transfer function, was characterized for rebuilding tunnel freestream spectrum from pitot-probe measurements. In addition, the pitot-probe transfer function was combined with hot-wire measurements to decompose freestream disturbances into the three Kovasznay's modes (i.e., acoustic, entropy, and vorticity modes) [31].

\section{A. Recovery of Freestream Disturbance Spectrum from Hypersonic Pitot-probe Measurements}

Researchers have previously attempted to account for the difference between a pitot-probe-measured spectrum and a freestream spectrum, including the so-called quasisteady analysis by Harvey et al. [32] and the unsteady analysis by Stainback and Wagner [33]. The quasisteady analysis was found to significantly underpredict the pitot probe fluctuations. The unsteady analysis of Stainback and Wagner only accounts for the sound-wave mode of freestream disturbances and yields a transfer function that is independent of frequency and probe geometry, thus cannot be used to relate the transducer-measured spectrum to the freestream spectrum.

To derive a transfer function for recovering the PSD of preshock static pressure, the research team of the University of Minnesota constructed a method to compute the transfer function by using DNS of flow around pitot probes with imposed freestream disturbances. Pitot probe geometries corresponding to recent characterizations of hypersonic facilities were considered at a variety of freestream conditions and disturbance types. All disturbance types are parameterized by a single fluctuating quantity $q^{\prime}$, which is pressure $p^{\prime}$ for acoustic disturbances and temperature $T^{\prime}$ for entropy disturbances. The imposed freestream disturbances were assumed to consist of $N$ discrete-frequency disturbances with the following form: 


$$
q^{\prime}(t)=\sum_{k=1}^{N} q_{k}^{\prime} \cos \left(\alpha_{k} x+\beta_{k} z-\omega_{k} t+\phi_{k}\right)
$$

in which $\alpha$ and $\beta$ are frequency-specific wavenumbers in the streamwise and vertical directions, respectively, defined by

$$
\begin{aligned}
& \alpha=\frac{\omega_{k} \cos \theta}{\bar{u} \cos \theta+\bar{c}} \\
& \beta=\frac{\omega_{k} \sin \theta}{\bar{u} \cos \theta+\bar{c}} .
\end{aligned}
$$

A subscript $k$ indicates a frequency-specific value, and $\phi_{k}$ is a randomly-selected phase. Mean flow quantities are denoted with an overline, and the free stream is assumed to be homogeneous in the spanwise direction. The normalized power spectral density is

$$
P S D_{k}=\frac{1}{2}\left(\frac{q_{k}^{\prime}}{\bar{q}}\right)^{2} .
$$

In a simulation, the pressure within the transducer radius is first area-averaged to yield the pressure timetrace measured by the transducer. The timetrace of area-averaged pressure is then Fourier-decomposed to yield the transducer-measured PSD. The transfer function $\chi$ is defined as the ratio of transducer-measured PSD to freestream PSD,

$$
\chi(f)=\frac{P S D_{t, k}}{P S D_{\infty, k}}
$$

in which a subscript $t$ indicates transducer-measured, and $\infty$ indicates free stream. DNS of flow around pitot probes were first conducted to characterize transfer functions for flow-parallel acoustic and entropic disturbances [34]. The transfer functions were found to exhibit a geometry-dependent resonance. Subsequent DNS were conducted for freestream acoustic disturbances inclined at $120^{\circ}$ relative to the bulk flow direction, with probe geometries and flow conditions corresponding to those in the Purdue BAM6QT facility. The incident angle of $120^{\circ}$ is similar to the angle of acoustic radiation from the nozzle-wall turbulent boundary layer (Fig. 7).

Figure 14 shows the DNS predictions for the transfer functions of angled freestream acoustic disturbances $\left(120^{\circ}\right.$ relative to the bulk flow direction) with and without area averaging and their comparison with flow-parallel slow acoustic disturbances. The transfer function exhibits a geometry-dependent resonance; the acoustic disturbance angle has a large effect on the transfer function, resulting in a smaller resonant frequency and a lower amplification factor for angled freestream acoustic disturbances than for flow-parallel freestream disturbances. Because of phase interference on the face of the pitot probe at high frequencies, the transfer function for angled acoustic disturbances shows a dependence on the area of the active sensing element, as indicated by the difference in face-averaged and stagnation-point transfer functions.

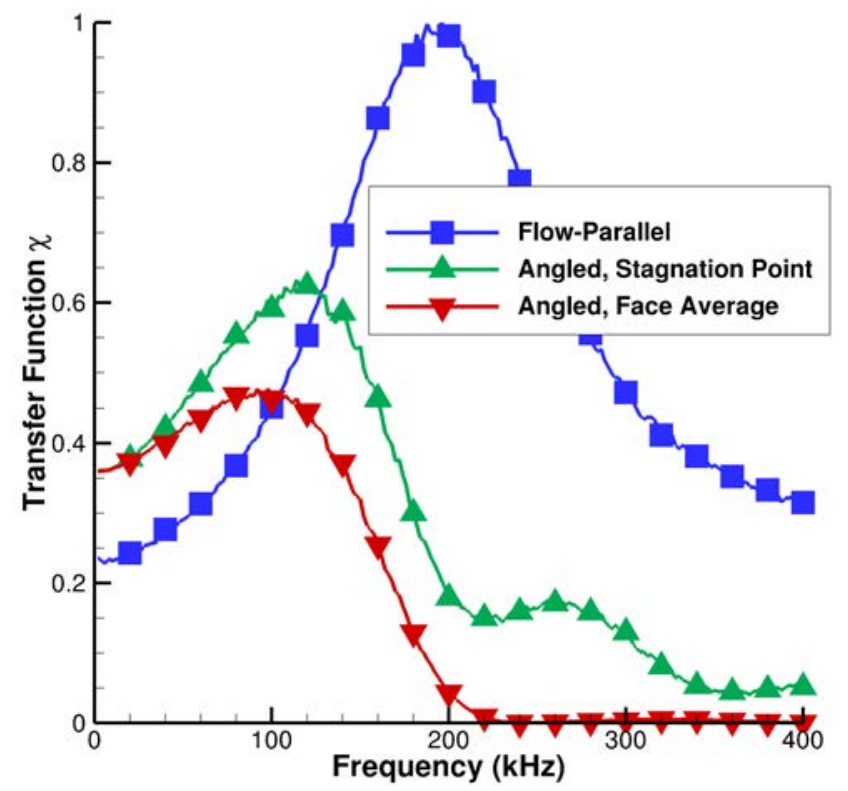

Figure 14. Transfer function for no-sleeve pitot probe geometry with angled freestream acoustic disturbances, flow-parallel slow acoustic disturbance included for comparison. 
The DNS predictions for transfer functions were compared to experimental measurements in the Purdue BAM6QT facility. The pitot probe geometries used in the experiments include four geometries; each uses the same sensing element with different flush-mounted shrouds to investigate the effect of probe geometry (Table 1). Figure 15 gives a picture of the probes used in the experiments. The experimental data were collected during two different weeks, referred to as Entry 1 and Entry 2. Given that the freestream disturbance spectra $P S D_{\infty}$ cannot be directly measured in an experiment, comparisons between DNS and experiments were done in terms of the ratio of transfer functions, $S$, with two different probe geometries:

$$
S_{a, b}(f) \equiv \frac{\chi_{a}}{\chi_{b}}=\frac{P S D_{a}}{P S D_{b}}
$$

The quantity $S$ is defined as the ratio between probes as shown, and is a frequency-dependent function. Figure 16 shows the ratio of each sleeved geometry to the no-sleeve geometry for both simulation and experiment. For example, $R_{\text {large }} \equiv S_{\text {large-sleeve,no-sleeve. }}$. The angled acoustic disturbances $\left(\theta=120^{\circ}\right)$ produce a much better match to experimental data than flow-parallel slow acoustic disturbances $\left(\theta=180^{\circ}\right)$. The results suggest that, with judiciously imposed freestream disturbances, the method of computing the transfer function using DNS of flow around pitot probes holds the potential to ultimately be used to replace the unsteady approach of Stainback and Wagner [33] for recovering freestream disturbance spectrum from stagnation pressure spectrum for hypersonic pitot probes.

\begin{tabular}{ccc} 
Probe Name & Transducer Radius & Total Radius \\
\hline No Sleeve & $0.83 \mathrm{~mm}$ & $0.83 \mathrm{~mm}$ \\
Small Sleeve & $0.83 \mathrm{~mm}$ & $1.50 \mathrm{~mm}$ \\
Medium Sleeve & $0.83 \mathrm{~mm}$ & $3.00 \mathrm{~mm}$ \\
Large Sleeve & $0.83 \mathrm{~mm}$ & $3.60 \mathrm{~mm}$
\end{tabular}

Table 1. Pitot probe geometries used to characterize freestream disturbance levels in the Purdue BAM6QT facility.

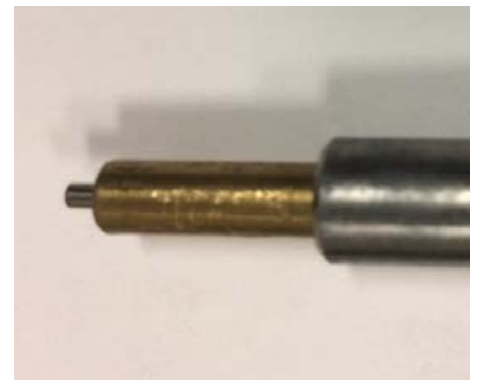

(a) No-sleeve case, Entry 1

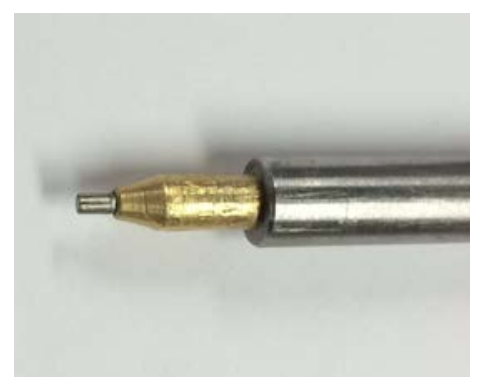

(c) No-sleeve case, Entry 2

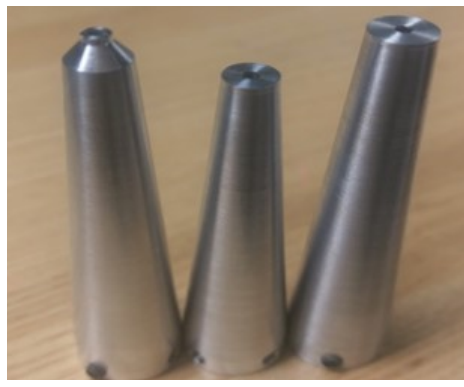

(b) Sleeves used in Entry 1

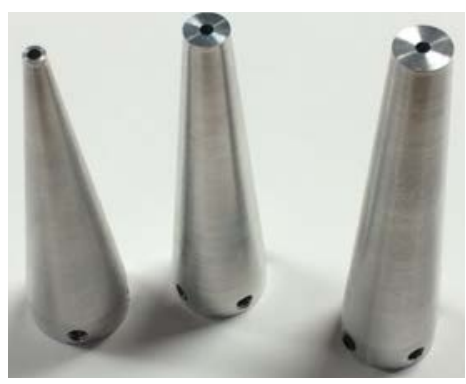

(d) Sleeves used in Entry 2

Figure 15. Pictures of pitot probe geometries used in the experiments. 


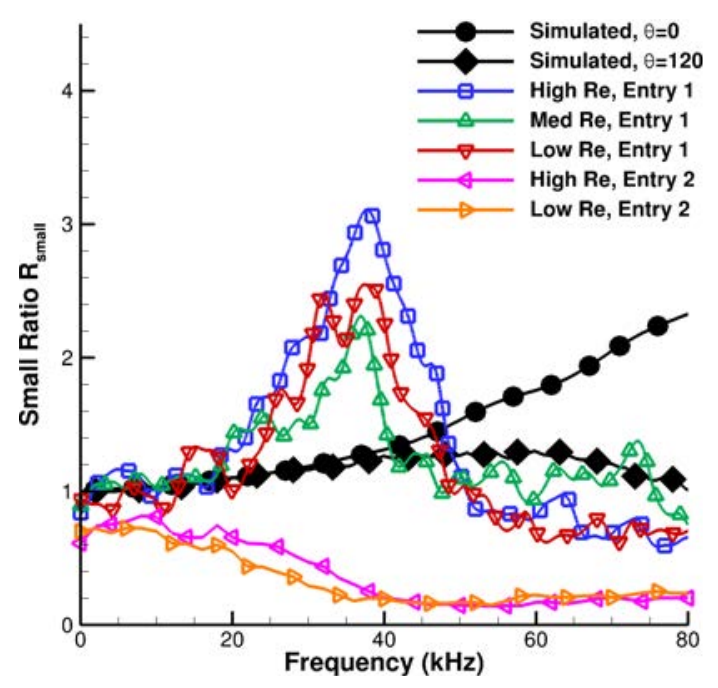

(a)

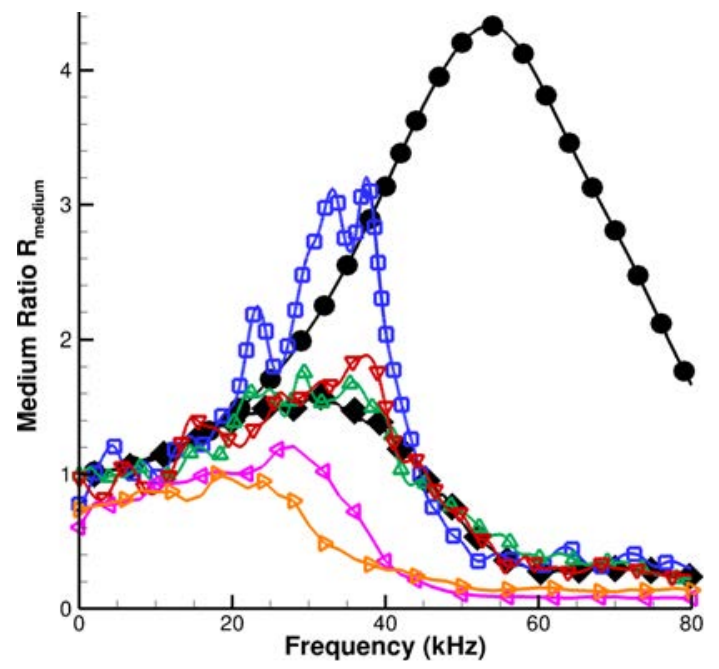

(b)

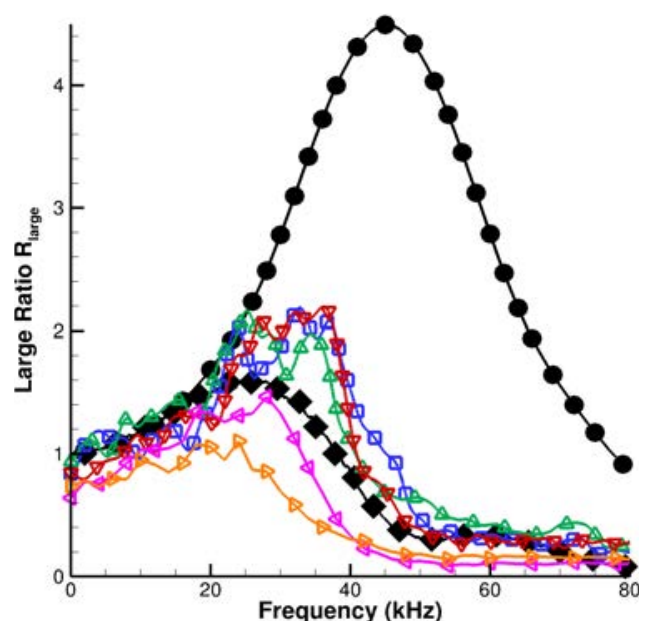

(c)

Figure 16. Ratio of probes $R$, comparing experiment to simulation. High Re: $R e \approx 11.5 \times$ $10^{6} / \mathrm{m} ;$ Med Re: $R e \approx 8.64 \times 10^{6} / \mathrm{m} ;$ Low $\operatorname{Re}: \operatorname{Re} \approx 3.01 \times 10^{6} / \mathrm{m}$.

\section{B. Kovasznay Modal Decomposition}

To better characterize the nature and origin of freestream disturbances in a high-speed wind tunnel, a new modal decomposition method was proposed to decompose wind-tunnel freestream disturbances into three modes of covarying physical properties: an acoustic or sound-wave mode (isentropic variation of pressure, density, and temperature as well as that of the coupled irrotational velocity field); an entropy mode (isobaric variation of entropy, density, and temperature, also mentioned as entropy or temperature spottiness); and a vorticity mode (variation of the solenoidal component of the velocity field, which is known as simple "turbulence" at low speeds). The concept and analytics of modal analysis goes back to Kovasznay [31] and Morkovin [7].

Early experimental investigations by Kovasznay [31], Morkovin [7] and Laufer [8], using hot-wire measurements, made use of the analytics to characterize supersonic wind tunnel flows. Later, Stainback and Wagner [33] conducted hot-wire and pitot probe measurements and compared resulting pressure fluctuations. Since hot-wire measurements yield fluctuations of static pressure and pitot probes measure total pressure, Stainback and Wagner [33] introduced an ansatz for the transfer function relating preshock static and post-shock total pressure fluctuations. Logan [35] proposed a new hot-wire modal analysis by considering separate static pressure fluctuation 
measurements using nonintrusive laser-induced fluorescence (LIF). The method of Logan was afterwards applied by Masutti et al. [19], Wu et al. [36], and Schilden et al. [37] among others using pitot probes and applying the transfer function by Stainback and Wagner [33]. Static pressure fluctuations due to vorticity and entropy fluctuations are neglected. They are generated during the interaction of the aforementioned disturbances with the shock wave upstream of the pitot probe [38, 39].

Another experimental and numerical approach using purely stagnation point probes (SPP) was introduced by Schilden et al. [37]. This modal decomposition method was based on total pressure and stagnation point heat flux fluctuations to decompose freestream disturbances via a sensitivity matrix containing transfer functions between the measured quantities and acoustic and entropy mode computed in DNS. Schilden and Schröder [40] showed that the proposed method is limited to very low Strouhal number of the incident disturbances, i.e., the heat flux probe has to be very small to perceive essential postshock entropy modes. At this point the hot-wire is a promising alternative to the rather large SPP since it is basically a very small heat flux probe. On the other hand, the transfer functions of Schilden and Schröder [40] or Chaudhry and Candler [34] relating post-shock total pressure to preshock acoustic and entropy disturbances could be used to replace the transfer function of Stainback and Wagner [33] in the hot-wire method. Therefore, the hot-wire and SPP modal decomposition methods can be merged.

The research teams of RWTH Aachen and TU Braunschweig proposed an improved technique for conducting combined modal analysis with data provided by hot-wire and pitot probe. Instead of using the transfer function of Stainback and Wagner [33], a sensitivity matrix containing the transfer functions between freestream disturbances and hot-wire and pitot data is used. The new combined modal decomposition method is described as follows:

\section{B.1. Data Acquisition}

The voltage applied to a hot-wire $E$ at constant temperature varies to compensate changes of heat loss due to flow disturbances. The heat loss is sensitive to mass flow $m=\rho u$ and total temperature $T_{0}$. The corresponding transfer functions $k_{m}$ and $k_{T_{0}}$ in

$$
\frac{d E}{E}=k_{m} \frac{d m}{m}+k_{T_{0}} \frac{d T_{0}}{T_{0}}
$$

are unknown. Voltage data postprocessing yields normalized r.m.s values $\langle\cdot\rangle$ (NRMS) of mass flow fluctuations $\langle m\rangle$, total temperature $\left\langle T_{0}\right\rangle$, and their correlation $R_{m, T_{0}}[31,36]$. For the later modal decomposition, the three quantities are combined to two hot-wire variables

$$
\begin{aligned}
& \left\langle H_{p, s}\right\rangle^{2}=\beta^{2}\langle m\rangle^{2}+\left\langle T_{0}\right\rangle^{2}-2 \beta R_{m, T_{0}} \\
& \left\langle H_{p, u}\right\rangle^{2}=\alpha^{2}\langle m\rangle^{2}+\left\langle T_{0}\right\rangle^{2}+2 \alpha R_{m, T_{0}}
\end{aligned}
$$

The coefficients $\alpha$ and $\beta$ are a function of the Mach number $M$ and the heat capacity ratio $\gamma$.

A pitot probe measures total pressure $p_{0}$, which contains acoustic pressure $d p$ and entropy fluctuations $d s$

$$
\frac{d p_{0}}{p_{0}}=\frac{r_{a c}}{\gamma} \frac{d p}{p}+r_{e n} \frac{d s}{C_{p}}
$$

The transfer functions $r_{a c}$ and $r_{e n}$ in Eq. 4 are formulated with respect to the preshock density fluctuations

$$
r_{i}=\frac{d p_{0}}{p_{0}} \frac{\rho_{\infty}}{d \rho_{i}} .
$$

The ratio of preshock average density and post-shock total pressure normalizes the transfer functions. Introducing NRMS values and neglecting any correlation between acoustic and entropy waves, Eq. 4 becomes

$$
\langle P\rangle^{2}=\left(\frac{r_{a c}}{\gamma}\right)\langle p\rangle^{2}+r_{e n}^{2}\langle s\rangle^{2} .
$$

$\langle P\rangle=\left\langle p_{0}\right\rangle$ denotes the data of the pitot pressure measurements, which are plugged into the modal decomposition method.

\section{B.2. Modal Decomposition}

Following the procedure of Logan [35], we start with the mass flow rate, the equation of state for ideal gas and the energy equation for inviscid flows, differentiate, and rewrite the system of equations for NRMS values to gain

$$
\begin{aligned}
& \left\langle H_{p, u}\right\rangle^{2}=\alpha^{2}\langle p\rangle^{2}+\theta^{2}\langle u\rangle^{2}+2 \alpha \theta R_{p, u} \\
& \left\langle H_{p, s}\right\rangle^{2}=\zeta^{2}\langle p\rangle^{2}+\theta^{2}\langle s\rangle^{2}
\end{aligned}
$$


under the aforementioned assumption of uncorrelated acoustic and entropy modes. The coefficients $\zeta$ and $\theta$ again depend on the Mach number and the heat capacity ratio. The remaining correlation term in Eq. 7 can be expressed as a perfectly correlated slow acoustic wave $R_{p, u}=-\langle p\rangle^{2} / \gamma M$. Finally, the velocity fluctuations $\langle u\rangle$ have to be split into the acoustic mode and vorticity mode contributions. Assuming no correlation between these disturbance types, $\langle u\rangle^{2}=\left\langle u_{a c}\right\rangle^{2}+\left\langle u_{v o}\right\rangle^{2}$ holds. The velocity fluctuations $\left\langle u_{a c}\right\rangle$ are again expressed by the pressure fluctuations of a slow acoustic wave. The complete system of equations of the modal decomposition can be written

$$
\begin{aligned}
& \left\langle H_{p, u}\right\rangle^{2}=\psi^{2}\langle p\rangle^{2}+\theta^{2}\left\langle u_{v o}\right\rangle^{2} \\
& \left\langle H_{p, s}\right\rangle^{2}=\zeta^{2}\langle p\rangle^{2}+\theta^{2}\langle s\rangle^{2} \\
& \langle P\rangle^{2}=\left(\frac{r_{a c}}{\gamma}\right)\langle p\rangle^{2}+r_{e n}^{2}\langle s\rangle^{2}
\end{aligned}
$$

In Eq. 9, $\psi$ is a function of Mach number and heat capacity ratio. The first two equations resemble the original equations of Logan [35]. The vorticity mode definition, i.e., velocity fluctuations $\langle u\rangle^{2}$ minus the contribution of the acoustic wave $\left\langle u_{a c}\right\rangle^{2}$, is incorporated. The last equation is extracted from the SPP method. The transfer functions $r_{a c}$ and $r_{e n}$ are computed in DNS and capture the frequency dependence present in SPP measurements. The consideration of the frequency dependence is crucial to apply Eqs. 9, 10, \& 11 to bandpass filtered data. In short, the modal analysis can be written $F_{w}=S F_{\infty}$ by using $F_{w}=\left(\left\langle H_{p, u}\right\rangle^{2},\left\langle H_{p, s}\right\rangle^{2},\langle P\rangle^{2}\right)^{T}$ and $F_{\infty}=\left(\langle p\rangle^{2},\langle s\rangle^{2},\left\langle u_{v o}\right\rangle^{2}\right)^{T} . S$ is the sensitivity matrix containing the transfer functions between freestream disturbances and hot-wire and pitot data.

\section{B.3. SPP and Combined Hot-wire Freestream Disturbances}

Experimental data of $F_{w}$, i.e., SPP data $\langle P\rangle$ and combined hot-wire data $\left\langle H_{p, u}\right\rangle$ and $\left\langle H_{p, s}\right\rangle$, were measured at three radial positions in HLB at varying unit-Reynolds numbers from $5 \times 10^{6} / \mathrm{m}$ to $18 \times 10^{6} / \mathrm{m}$. The measurement locations are on the centerline, and $50 \mathrm{~mm}$ and $100 \mathrm{~mm}$ off the centerline. In Fig. 17, the left-hand sides of Eqs. 10 and 11 are shown. According to the final system of equations of the modal decomposition only the acoustic and entropy mode contribute to $\left\langle H_{p, s}\right\rangle$ and $\langle P\rangle$. Bandpass filtered data are shown in Fig. 17a to illustrate the frequency effect. The acoustic and entropy noise in the wind tunnel leads to different trends in $\left\langle H_{p, s}\right\rangle$ and $\langle P\rangle$. Whereas the total pressure decays exponentially with increasing frequency, the combined hot-wire variable shows a spectral decay of type $\sim f^{-m}$ using a log-log scale (not shown here). The centerline data always contain the most intense disturbances. The dependence on unit Reynolds number in Fig. $17 \mathrm{~b}$ is very small at a frequency of $f=102 \mathrm{kHz}$. To apply the previously derived modal decomposition method to the measured freestream disturbance variables $F_{w}$, we want to determine all transfer functions in the sensitivity matrix $S$, thus accounting for frequency dependence and the inclination of incident acoustic waves.

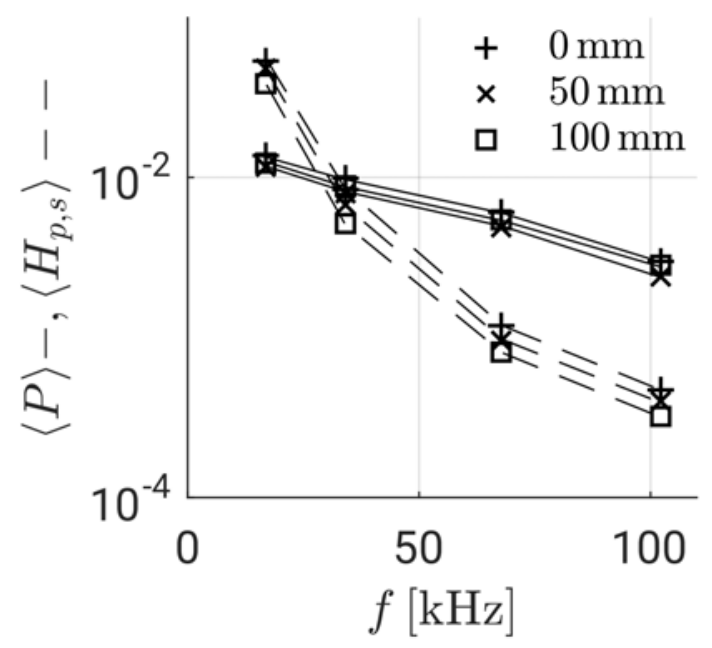

(a)

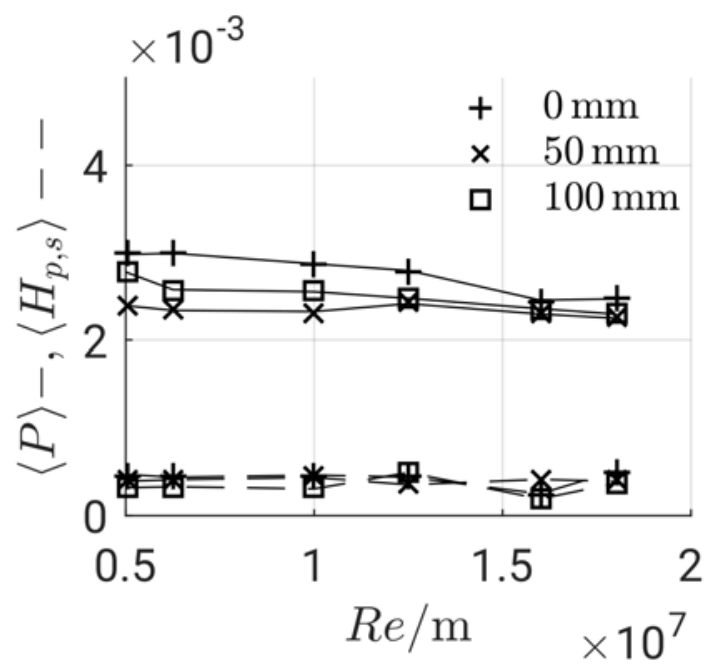

(b)

Figure 17. Experimental bandpass filtered hot-wire $\left\langle H_{p, s}\right\rangle$ and SPP $\langle P\rangle$ data from the Hypersonic Ludwieg Tube of the Technical University Braunschweig. The dashed line represents the hot-wire data, the solid line the SPP data. The symbols indicate the distance to the centerline of the wind tunnel: a) effect of frequency at unit Reynolds number $R e=5 \times 10^{6} / \mathrm{m}$; b) effect of unit Reynolds number at $f=102 \mathrm{kHz}$. 


\section{Summary and Conclusion}

This paper outlines some of the coordinated experimental and numerical work by the NATO STO AVT-240 specialists group that yielded freestream disturbance data that is physically relevant for transition processes in hypersonic flows. New freestream disturbance measurements with fast-response pressure transducers mounted on a variety of probes were performed and provided spectral data up to the high frequencies of relevance to second-mode instabilities. DNS of high-speed turbulent boundary layers and their acoustic radiation were conducted to allow direct computation of the stochastic disturbance field within the free stream. The DNS studies of acoustic radiation have overcome difficulties in experimental measurements and provided access to both flow and acoustic quantities that are difficult to obtain otherwise, including the acoustic disturbance angle, the propagation speed, and the acoustic sources. DNS of flow around a pitot probe were performed to cover the transfer of disturbances to the detailed location of the flow sensor as mounted in the probe, resulting in the recovery of freestream disturbance spectrum from the transducer-measured spectrum. In particular, the DNS study showed that the acoustic angle has a large effect on the pitot-probe transfer function, and the results for angled disturbances, with an incident acoustic angle similar to that of acoustic radiation from the nozzle wall, agreed much more closely with the experimental measurements. A new modal decomposition method was proposed to decompose hot-wire and pitot-probe measurements of freestream disturbances into the three Kovasznay modes (i.e., acoustic, entropy, and vorticity modes). By replacing the pitot-probe transfer function by Stainback and Wagner [33] with the DNS-predicted pitotprobe transfer function by Schilden and Schröder [40], the new combined modal decomposition method can yield r.m.s. values of modal amplitudes within a selected frequency band.

The joint effort of the specialists group has resulted in an improved knowledge base pertaining to the nature and spectral contents of wind tunnel freestream disturbances. The new high-frequency disturbance data have been used in formulating correlations for the spectra of initial disturbances in hypersonic boundary layers [25]. The data may be used for feeding freestream disturbances into advanced DNS transition computations, thus opening the door to holistic simulations of transition in a natural disturbance environment.

\section{Acknowledgments}

This work was sponsored by the Air Force Office of Scientific Research (under grants FA9550-14-1-0170, FA9550-17-1-0250, and FA9550-12-1-0167). Computational resources were provided by the DoD High Performance Computing Modernization Program, the NASA Advanced Supercomputing Division, and the NSF PRAC program (NSF ACI-1640865). Eric Marineau would like to acknowledge the Test Resource Management Center T\&E / S\&T HSST program for their funding as part of the Center of Testing Excellence. The views and conclusions contained herein are those of the authors and should not be interpreted as necessarily representing the official policies or endorsements, either expressed or implied, of the funding agencies or the U.S. Government. Sandia National Laboratories is a multi-mission laboratory managed and operated by National Technology and Engineering Solutions of Sandia, LLC., a wholly owned subsidiary of Honeywell International, Inc., for the U.S. Department of Energy's National Nuclear Security Administration under contract DE-NA0003525.

\section{References}

[1] Schneider, S. P., "Development of Hypersonic Quiet Tunnels," Journal of Spacecraft and Rockets, Vol. 45, No. 4, 2008, pp. 641-664.

[2] Beckwith, I. E., and Miller, C. G., "Aerothermodynamics and Transition in High-Speed Wind Tunnels at NASA Langley," Annual Review of Fluid Mechanics, Vol. 22, 1990, pp. 419-439.

[3] Zhang, C., Duan, L., and Choudhari, M. M., "Effect of wall cooling on boundary-layer-induced pressure fluctuations at Mach 6," Journal of Fluid Mechanics, Vol. 822, 2017, pp. 5-30.

[4] Huang, J., Duan, L., and Choudhari, M. M., "Direct Numerical Simulation of Acoustic Noise Generation from the Nozzle Wall of a Hypersonic Wind Tunnel," AIAA Paper 2017-3631, 2017.

[5] Zhang, C., Duan, L., and Choudhari, M. M., "Acoustic Radiation from a Mach 14 Turbulent Boundary layer," AIAA Paper 2016-0048, 2016.

[6] Phillips, O. M., "On the generation of sound by supersonic turbulent shear layers," Journal of Fluid Mechanics, Vol. 9, No. 1, 1960, pp. 1-28.

[7] Morkovin, M. V., "On Transition Experiments at Moderate Supersonic Speeds," Journal of the Aeronautical Sciences, Vol. 24, No. 7, 1957, pp. 480-486.

[8] Laufer, J., "Aerodynamic Noise in Supersonic Wind Tunnels," Journal of the Aerospace Sciences, Vol. 28, No. 9, 1961, pp. 685-692.

[9] Laufer, J., "Some statistical properties of the pressure field radiated by a turbulent boundary layer," Physics of Fluids, Vol. 7, No. 8, 1964, pp. 1191-1197. 
[10] Pate, S. R., "Measurements and Correlations of Transition Reynolds Numbers on Sharp Slender Cones at High Speeds," AIAA Journal, Vol. 9, No. 6, 1971, pp. 1082-1090.

[11] Stetson, K., "Nosetip bluntness effects on cone frustum boundary layer transition in hypersonic flow," AIAA Paper 1983-1763, 1983.

[12] Williams, J. E. F., "On the development of Mach waves radiated by small disturbances," Journal of Fluid Mechanics, Vol. 22, No. 1, 1965, pp. 49-55.

[13] Schneider, S. P., "Effects of High-Speed Tunnel Noise on Laminar-Turbulent Transition," Journal of Spacecraft and Rockets, Vol. 38, No. 323-333, 2001.

[14] Marineau, E. C., Moraru, G. C., Lewis, D. R., Norris, J. D., Lafferty, J. F., and Johnson, H. B., "Investigation of Mach 10 Boundary Layer Stability of Sharp Cones at Angle-of-Attack, Part 1: Experiments," AIAA Paper 2015-1737, 2015.

[15] Kendall, J. M., "Supersonic boundary layer transition studies," JPL Space Programs Summary, Vol. 3, 1970, pp. 4347.

[16] Donaldson, J., and Coulter, S., "A review of free-stream flow fluctuation and steady-state flow quality measurements in the AEDC/VKF Supersonic Tunnel A and Hypersonic Tunnel B," AIAA Paper 1995-6137, 1995.

[17] Lafferty, J., and Norris, J., "Measurements of Fluctuating Pitot Pressure, "Tunnel Noise," in the AEDC Hypervelocity Wind Tunnel No. 9," AIAA Paper 2017-1678, 2007.

[18] Bounitch, A., Lewis, D., and Lafferty, J., "Improved Measurements of "Tunnel Noise" Pressure Fluctuations in the AEDC Hypervelocity Wind Tunnel No. 9," AIAA Paper 2011-1200, 2011.

[19] Masutti, D., Spinosa, E., Chazot, O., and Carbonaro, M., "Disturbance Level Characterization of a Hypersonic Blowdown Facility," AIAA Journal, Vol. 50, No. 12, 2012, pp. 2720-2730.

[20] Laufer, J. "Sound radiation from a turbulent boundary layer," Proceedings of the Marseille Conference on Turbulence. CNRS Report 108. 1962.

[21] Fujii, K., "Experiment of the Two-Dimensional Roughness Effect on Hypersonic Boundary-Layer Transition," Journal of Spacecraft and Rockets, Vol. 43, No. 4, 2006, pp. 731-738.

[22] Parziale, N. J., Shepherd, J. E., and Hornung, H. G., "Free-stream density perturbations in a reflected-shock tunnel," Experiments in Fluids, Vol. 55, No. 2, 2014, pp. 1-10.

[23] Pate, S. R., "Dominance of Radiated Aerodynamic Noise on Boundary-Layer Transition in Supersonic-Hypersonic Wind Tunnels," Technical Report AEDC-TR-77-107, 1978.

[24] Pate, S. R., "Effects of wind tunnel disturbances on boundary-layer transition with emphasis on radiated noise," AIAA Paper 1980-0431, 1980.

[25] Marineau, E. C., "Prediction Methodology for Second-Mode-Dominated Boundary-Layer Transition in Wind Tunnels," AIAA Journal, Vol. 55, No. 2, 2017, pp. 484-499.

[26] Duan, L., Choudhari, Meelan M., and Wu, M., "Numerical study of acoustic radiation due to a supersonic turbulent boundary layer," Journal of Fluid Mechanics, Vol. 746, 2014, pp. 165-192.

[27] Duan, L., Choudhari, M. M., and Zhang, C., "Pressure fluctuations induced by a hypersonic turbulent boundary layer," Journal of Fluid Mechanics, Vol. 804, 2016, pp. 578-607.

[28] Duan, L., and Choudhari, M. M., "Analysis of Numerical Simulation Database for Acoustic Radiation from HighSpeed Turbulent Boundary Layers," AIAA Paper 2014-2912, 2014.

[29] Zhang, C., and Duan, L., "Acoustic Radiation from High-Speed Turbulent Boundary Layers in a Tunnel-like Environment," AIAA Paper 2015-0836, 2015.

[30] Huang, J., Zhang, C., Duan, L., and Choudhari, M. M., "Direct Numerical Simulation of Hypersonic Turbulent Boundary Layers inside an Axisymmetric Nozzle," AIAA Paper 2017-0067, 2017.

[31] Kovasznay, L. S. G., "Turbulence in Supersonic Flow," Journal of the Aeronautical Sciences, Vol. 20, No. 10, 1953, pp. 657-674.

[32] Harvey, W. D., Bushnell, D. M., and Beckwith, I. E., "Fluctuating properties of turbulent boundary layers for Mach numbers up to 9 " NASA TN D-5496, 1969.

[33] Stainback, P. C., and Wagner, R. D., "A comparison of disturbance levels measured in hypersonic tunnels using a hotwire anemometer and a pitot pressure probe," AIAA Paper 1972-1003, 1972.

[34] Chaudhry, R. S., and Candler, G. V., "Computing Measured Spectra from Hypersonic Pitot Probes with Flow-Parallel Freestream Disturbances," AIAA Journal, 2017, pp. 1-12.

[35] Logan, P., "Modal Analysis of Hot-wire Measurements in Supersonic Turbulence," AIAA Paper 1988-0423, 1988.

[36] Wu, J., Zamre, P., and Radespiel, R., "Flow quality experiment in a tandem nozzle wind tunnel at Mach 3," Experiments in Fluids, Vol. 56, No. 1, 2015, pp. 20-37.

[37] Schilden, T., Schröder, W., Ali, S. R. C., Schreyer, A.-M., Wu, J., and Radespiel, R., "Analysis of acoustic and entropy disturbances in a hypersonic wind tunnel," Physics of Fluids, Vol. 28, No. 5, 2016, p. 056104.

[38] McKenzie, J. F., "Interaction of Linear Waves with Oblique Shock Waves," Physics of Fluids, Vol. 11, No. 11, 1968, pp. 2350-2362.

[39] Anyiwo, J. C., and Bushnell, D. M., "Turbulence amplification in shock-wave boundary-layer interaction," $A I A A$ Journal, Vol. 20, No. 7, 1982, pp. 893-899.

[40] Schilden, T., and Schröder, W., "Numerical analysis of high speed wind tunnel flow disturbance measurements using stagnation point probes," Journal of Fluid Mechanics, Vol. 833, 2017, pp. 247-273. 